\title{
Estimating costs and health outcomes of publicly funded tick-born encephalitis vaccination: A cost- effectiveness analysis
}

J ad Shedrawy, Martin Henriksson, Maria-Pia Hergens and H. Helena Askling

The self-archived postprint version of this journal article is available at Linköping University Institutional Repository (DiVA):

http:// urn.kb.se/ resolve?urn=urn:nbn:se:liu:diva-153831

N.B.: When citing this work, cite the original publication.

Shedrawy, J., Henriksson, M., Hergens, M., Askling, H. H., (2018), Estimating costs and health outcomes of publicly funded tick-born encephalitis vaccination: A cost-effectiveness analysis, Vaccine, 36(50), 7659-7665. https:// doi.org/ 10.1016/j.vaccine.2018.10.086

Original publication available at:

https:/ / doi.org/ 10.1016/j.vaccine.2018.10.086

Copyright: Elsevier (12 months)

http:// www.elsevier.com/

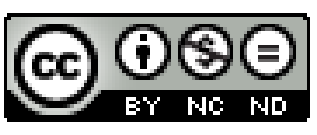


Abstract

\section{BACKGROUND}

The number of notified cases of Tick-Borne Encephalitis (TBE) in Sweden has been increasing the past years despite the increased use of TBE-vaccine not subsidized by the healthcare system. Stockholm County is a high endemic area and an earlier study has shown that low-income households have lower vaccination coverage even when they are at high risk. This paper aims to determine the cost-effectiveness of a publicly funded TBE vaccination program in Stockholm.

\section{METHODS}

In three different cohorts with individuals aged 3, 40 or 50 years, long-term costs and health outcomes of an out-of-pocket strategy (53\% of the cohort is vaccinated on their own expenses) and a structured vaccination program (full cohort is vaccinated covered by the publicly funded health care system), were estimated using a Markov model. The Markov model predicts the costs and effects (in term of Quality-adjusted Life Years (QALYs) over a lifetime horizon using a third-party healthcare payer perspective. The primary results are presented as an incremental cost effectiveness ratio (ICER) indicating the additional cost required to achieve one additional QALY with the structured vaccination program.

\section{RESULTS}

The results show that the structured vaccination program is associated with a gain in QALYs and increased costs compared with an out-of-pocket strategy. The calculated ICERs were 27 761, 99527 and 160827 SEK/QALY in cohorts of age 3, 40 and 50, respectively. The sensitivity analyses showed that the results are robust when varying different parameters.

\section{CONCLUSION}


Given the setting of Stockholm county, this analysis shows a cost per QALY of a free vaccinations program, especially for children of 3 years old, below generally acceptable costeffectiveness thresholds in Sweden. 


\section{Background}

\section{TBE infection and prevention}

Tick-borne Encephalitis (TBE) is caused by a virus from the family Flaviviridae. TBE-virus exists in three main subtypes; European, Siberian and Far-Eastern, and transmission to humans occurs mainly via infected tick-bites. [1] TBE is reported throughout Russia, Baltics, Scandinavia and eastern and central Europe. During the last years there has been a change in the epidemiology of the disease, with new risk areas in Western Europe. [2] Recently a first case of TBE with documented transmission was reported in the Netherlands [3] and the Siberian virus type has been reported from Finland [4] The preferred tick for this virus type, Ixodes persulcatus, has been found in northern Sweden. [5]

TBE symptoms range from mild fever to severe meningoencephalitis, paresis and unconsciousness. The disease from European subtype is typically biphasic, after an incubation period of 4-28 days, the first febrile stage lasts around four days (1-8) followed by an asymptomatic stage of 8 days (1-33 days). This is then followed typically by an acute meningoencephalitis lasting 1-3 weeks. Mortality is $0.5-2 \%$ depending on age. Neurological sequelae is reported in $30-40 \%$ of cases, ranging from concentration disabilities to tremor and paresis. [1,6-12] In Europe two inactivated vaccines are presently registered, FSME-immune (available in Sweden since 1980s, Pfizer) and Encepur (GSK). The immunogenicity of any of those vaccines is 95-100\%.[13-15] Vaccine effectiveness has been assessed in Austria, where vaccination is recommended and partly subsidized, showing 96-99 \% field effectiveness for regularly vaccinated persons.[16] Antibody response has been shown to be decreased in the older population and vaccination failures, i.e. disease despite documented vaccination, are more common among elderly and immunosuppressed individuals. [17-19] 


\section{TBE and vaccination in Sweden}

In Sweden, TBE is a notifiable disease according to the Communicable Diseases Act and the notification is based on both clinical and laboratory reporting. There has been a steadily increasing trend in number of cases the recent 10 years; [20] accompanied with an increase in TBE-vaccines sales according to the vaccine manufactures.[21] Current trend indicates that the risk-areas are expanding and therefore an increasing demand for vaccination. There are no national TBE-vaccination recommendations but instead an annual consensus meeting with Medical County Officers in cooperation with the Public Health Agency provide guidance. According to the 2018 meeting vaccination recommendations are as follows: two priming doses with one month interval for individuals up to age 49; three priming doses within four months for individuals from age 50 or immunosuppressed at any age; thereafter, for all groups, a booster dose before next tick season 5-12 months later; after three year another booster dose followed by a booster dose every five year independent of age. [22] All individuals pay for the vaccine themselves, with an out-of-pocket burden of approximately 30/35 Euros for children/adults, per vaccine dose.

Lacking a national vaccination register, in order to investigate TBE-vaccination coverage, a recent study performed in Stockholm county revealed that 53 percent of inhabitants aged 1 year or older were vaccinated a least with one dose. The cost of the vaccine and fear of adverse events was reported as the most common reasons for not vaccinating. Furthermore, low household income was associated with non-vaccination, despite exposure to risk-areas outdoors. The incidence of TBE in the non-vaccinated population of Stockholm county was estimated at 8-12/100 000, with the highest incidence 12/100 000 in the group of elderly above 60 years old. [23] These findings indicate that Stockholm county is a high-risk area comparable to other high-risk areas such as the Baltics and Austria where vaccination has been implemented and subsidized. The current situation raises the question whether a publicly 
funded vaccination program would increase vaccination rates and lower the risk of TBErelated problems. However, such a program would compete with other resources in the healthcare sector and therefore it is important to establish the long-term costs and health outcomes of a local TBE vaccination strategy in order to understand if funding of a TBE vaccination program yield better health outcomes at a reasonable cost.

Aim

The aim of this study was to determine the cost-effectiveness of a publicly funded TBE vaccination program in a high-risk area of Sweden. 


\section{METHODS}

\section{Cost-effectiveness analysis overview}

Three different cohorts were subject to evaluation; individuals aged 3, 40 or 50 years, respectively. Long-term costs and health outcomes were estimated for two broad management strategies for each of these start ages:

1- Out-of-pocket vaccination: no structured vaccination program exists and $53 \%$ of the cohort is vaccinated on their own expenses (aligned with the latest data) at the start of the model; with no booster doses accounted for.

2- Structured vaccination program: individuals receive three doses of vaccine during the first year (four doses for the cohort of 50 years old), a booster dose after three years followed by booster doses (every 10 years for the three years old cohort and every 5 years for the other two cohorts) until the age of 80.

The choice of the 3 different cohorts was motivated by both immunological and vaccinological reasons given that the optimal vaccination age in terms of immunological priming is after 2-3 years old and before teenage, which also corresponds to the age when children are best reached in pre-school or schools. This motivates the 3-year old age start. Furthermore, by the age of 40 immunological response to vaccination is starting to wane. In Sweden an extra priming dose has recently been recommended when starting to vaccinate against TBE after age 50, and up until now this was limited to individuals aged above 60 . Therefore, these two older age cohorts were chosen. Furthermore, different age cohorts allow us to investigate the impact of morbidity and mortality burden due to age on costeffectiveness of a structured vaccination program. 
Health outcomes in terms of quality-adjusted life years (QALYs), incorporating both length of life and quality of life [24] as well as health care costs were estimated by employing decisionanalytic modelling. Clinical and epidemiological inputs were derived from different sources and included in the model. Extensive sensitivity analyses were undertaken in order to assess uncertainty in the estimated costs and health outcomes. The analysis primarily takes a thirdparty payer perspective, considering only costs that fall on the health care sector without accounting for any out-of-pocket costs paid by the individuals. Costs and health outcomes were discounted by 3 per cent per annum according to current Swedish guidelines.[25] The primary results are presented as an incremental cost effectiveness ratio (ICER) which should be interpreted as the additional cost required to achieve one additional QALY with a structured vaccination program compared with the out of pocket strategy. [24]

\section{Decision analytic model}

To model the progression of TBE with and without a vaccination program in cohorts of healthy individuals a Markov structure was developed in Microsoft Excel (version 16.14.0). In the Markov model, individuals reside in one of a set of mutually exclusive states and can move between these states according to transition probabilities that reflect the risk of TBE, disease progression and mortality. Each state in the model is associated with a healthcare cost and a health outcome (QALYs).

The Markov model was designed with 8 health states (Figure 1) in which individuals could reside for one year (annual Markov cycles) before a potential transition to another state. The "healthy" state corresponds to individuals with no TBE infection. Individuals infected with TBE move from the "healthy” state to either “Die TBE” or "Survive TBE” depending on 
whether they survived the TBE during the incident year. Individuals stay in "Survive TBE" for one cycle (year) only and then move to either the "mild”, "moderate”, "severe” or "recovered" state depending on the severity of the sequelae. It was assumed that individuals stayed in the "mild”, “moderate”, "severe” or "Recovered” state for the remainder of the analysis. In any cycle, individuals are at risk of dying and move to the "Dead" state.

\section{Data}

The following section describes data sources and inputs into the decision-analytic model. All data inputs are summarized in Table 1.

\section{TBE incidence and long term outcomes}

The incidence of TBE infection by age group is derived from notification data from Stockholm County and a vaccination coverage study from the same area [23]. The probabilities of events after the incidence year were derived from several follow-up studies on TBE-infection.[1,6-12]

\section{Vaccine efficacy}

The vaccine efficacy was estimated to be $98.7 \% .[13-15]$ The vaccine efficacy was assumed to last 10 years after the fourth dose in children and 5 years in the adult cohorts. [26,27] Both vaccines available in Sweden are considered equal immunogenic and interchangeable.[28]

\section{Mortality}

The probabilities of dying due to TBE in the incidence year were derived from clinical follow up studies on TBE. [1,6-12] There is no available data regarding the increased risk of death from TBE after the incidence year and therefore it was assumed that these probabilities are the 
same as for the incident year. As patients with severe sequelae have a higher risk of dying from other causes like infections and respiratory insufficiency this appears to be a plausible assumption. The importance of this assumption for the final results was further investigated in sensitivity scenarios. As patients continuously grow older by each year of analysis in the model, all mortality rates are adjusted upward annually using age-specific mortality from the general population. [29]

\section{Costs}

The cost of the TBE vaccine, first dose and booster dose, was allocated to each cycle the vaccine was given. The cost was derived from the Stockholm county council regular tender process on vaccines in 2017 (Table 1). [30] The costs of hospitalizations and policlinic visits to multidisciplinary clinics during the incident year were derived from local cost per TBEpatient administrative data bases (ValDATA). The data bases include monthly-updated records of publicly-funded health care in Stockholm and was used to calculate an average cost associated with the incident years. As all Swedish patients are given a specific diagnosis code as soon as they are entering the health-care system these cost were specifically derived only from patients with a diagnosis of TBE, and should therefore primarily be associated with TBE disease. [31] The average costs of hospitalization in the subsequent years were taken from data on patients suffering from stroke in Sweden due to lack of any data from TBE patients.[32]

\section{Health related quality of life}

Health related quality of life (HRQoL) weights to adjust life-expectancy for the quality of life were estimated by age from the general population in Sweden for healthy individuals. [33] For TBE patients, decrements in HRQoL were assigned to the sequelae health states (Table 
1). The decrements were derived from a HRQoL study among Stroke patients in Denmark due to similarity in the clinical representation of both diseases at different stages. [34] It was assumed that patients in the "recover" health state have the same HRQoL as the general population. There is no available data regarding HRQoL during the incidence year of TBE and therefore it was assumed that the health state "survive TBE" is associated with the same HRQoL as the moderate sequelae.

\section{Analysis}

A lifetime time horizon was adopted in the analysis of the model, implying that all patients had transitioned to the dead state at the termination of analysis. [35] Cumulative costs and health outcomes from all Markov cycles were calculated for the structured vaccination program and the out-of-pocket strategy cohorts. The differences in the costs and health outcome between the two comparators were calculated and presented an ICER which is the additional costs associated with one extra unit of the health outcome by employing a structured vaccination program compared with no program.

\section{Sensitivity analysis}

For particular model parameters that has been assumed or extrapolated, a range of values were tested in one-way sensitivity analyses to investigate the importance of the parameter for the cost-effectiveness results and the parameters with the most influence on the ICER are presented in the results section. In addition, a probabilistic sensitivity analysis (PSA) was performed where all parameters associated with statistical uncertainty are varied simultaneously by sampling from probability distributions reflecting the quantity and quality of evidence of the parameters. Cost parameters followed a gamma distribution while probabilities of events and QALY weights followed a beta distribution in the probabilistic 
analysis. By running 1000 simulations, this exercise provides a joint distribution of incremental costs and outcomes associated with the vaccination strategy that can be summarized in a cost-effectiveness acceptability curve. The curve illustrates the probability of the vaccination strategy being cost-effective at different threshold values of costeffectiveness. [35] 


\section{Results}

\section{Base-case analysis}

The results of the base-case analysis show that the structured vaccination program is associated with an improvement in QALYs and increased costs compared with a no vaccination strategy for all cohorts with ICERs of 27761 , 99527 and 169827 SEK/QALY for the cohorts of 3, 40 and 50-year olds, respectively (Table 2). In younger cohorts, incremental costs are lower and the gain in QALYs higher implying a more favourable ICER compared with older cohorts.

\section{Sensitivity analysis}

The one-way sensitivity analysis (figure 2) shows that the majority of the parameters tested do not influence the ICER to an extent that change the cost-effectiveness interpretation. The cost of hospitalization in subsequent years for severe cases seems to have the biggest influence where the ICER can vary from around 15000 to 65000 SEK/QALY when using cost values of 90000 SEK and 200000 SEK respectively.

The cost effectiveness acceptability curve (figure 3) shows that at any cost-effectiveness threshold the probability of vaccination being cost-effective is higher for the cohort with the start age of 3 compared with the other groups. For example, for that cohort, the probability of the vaccination program being cost effective is around $80 \%$ using a cost-effectiveness threshold of 50000 SEK/QALY. 


\section{Discussion}

This study shows the cost per QALY of a structured vaccination program compared with an out-of-pocket program is 30 000SEK, 100 000SEK and 160 000SEK for three, forty and fiftyyear olds, respectively. Although there is no explicit cost-effectiveness threshold in Sweden, decisions made by the pharmaceutical benefits agency regarding the reimbursement of pharmaceuticals indicate that this value may be somewhere between 250 000SEK and 1000 000SEK, depending on the severity of the disease and other decision modifying factors. With this benchmark, a structured vaccination program appears cost-effective, in particular for younger individuals. [23]

In 1996, a crude estimation was made concerning the cost-effectiveness of TBE-vaccination in the Stockholm area and it was calculated that in view of a maximum TBE- incidence of 3.4/100,000 and the costs of vaccination, mass vaccination would be an unrealistic alternative. [6] From our own data we can now, more than 20 years later, report a much higher incidence in the unvaccinated population. [23] An official report, although not published in a peer reviewed paper, from another TBE-endemic Swedish county, Sörmland, [36] showed a very high cost per QALY of vaccinating all individuals when estimating a vaccination coverage of $25 \%$ and an incidence of 0.00035 . In 2015 an analysis of a randomly selected survey in Sweden projected that a subsidy making TBE vaccines free of charge could increase the vaccination rate in TBE risk areas to around 78\%, with a larger effect on low-income households [21]. From the European perspective, a Slovenian study showed a cost-effective result of vaccinating all individuals from the age of 18. [37] However the costs and 
assumptions used cannot be transferred to the Swedish setting making any comparison with our results highly uncertain.

This study has been performed from the third-party payer perspective only. However, TBE can be associated with a high productivity loss, mainly for patients with moderate or severe sequelae. Considering consequences falling outside the health care sector may thus be in favour of a vaccination program. In addition, the out-of-pocket costs represented by the cost of vaccine paid by individuals in the "out of pocket” strategy may have a positive impact on individual's private consumption that is not included in the analysis from a health care perspective.

\section{Limitations}

Due to lack of data from TBE patients, data from stroke studies (HRQoL and costs of subsequent years) was used which may compromise the accuracy of the results. Clinical experts appear to rely on the similarity of the consequences of the sequelae between these two diseases providing some support for our extrapolation of data. In addition, one-way sensitivity analysis was performed on these parameters showing a modest impact on the estimated ICERs even when employing extreme values.

Concerning the vaccine efficacy we have estimated quite a high immunogenicity of $98.7 \%$ for the whole group, even if it is well known that elderly individuals and immunosuppressed patients have a weaker vaccinations response and risk of vaccination failure. [17-19] Thus the cost-effectiveness in the older group might be overestimated.

Another potential overestimation is related to the adherence to the vaccination program as the present analysis assumes that everyone in the vaccinated cohort will adhere to all the booster doses, which does not reflect the drop-out that occurs in reality. On the other hand it is 
assumed in the out-of-pocket strategy that individuals are protected for a certain period of time with only one dose of the vaccine, which is an overestimation of the efficacy of the comparator strategy.

\section{Conclusion}

Given the setting of Stockholm county, this analysis shows a cost per QALY of a free vaccinations program, especially for children of 3 years old, below generally acceptable costeffectiveness thresholds in Sweden. We assume our findings might be extrapolated to other high endemic areas in Europe with similar health care costs and TBE-vaccination coverage.

\section{Conflict of interest}

The authors declare no conflict of interest

\section{Funding}

This research did not receive any specific grant from funding agencies in the public, commercial, or not-for-profit sectors. 


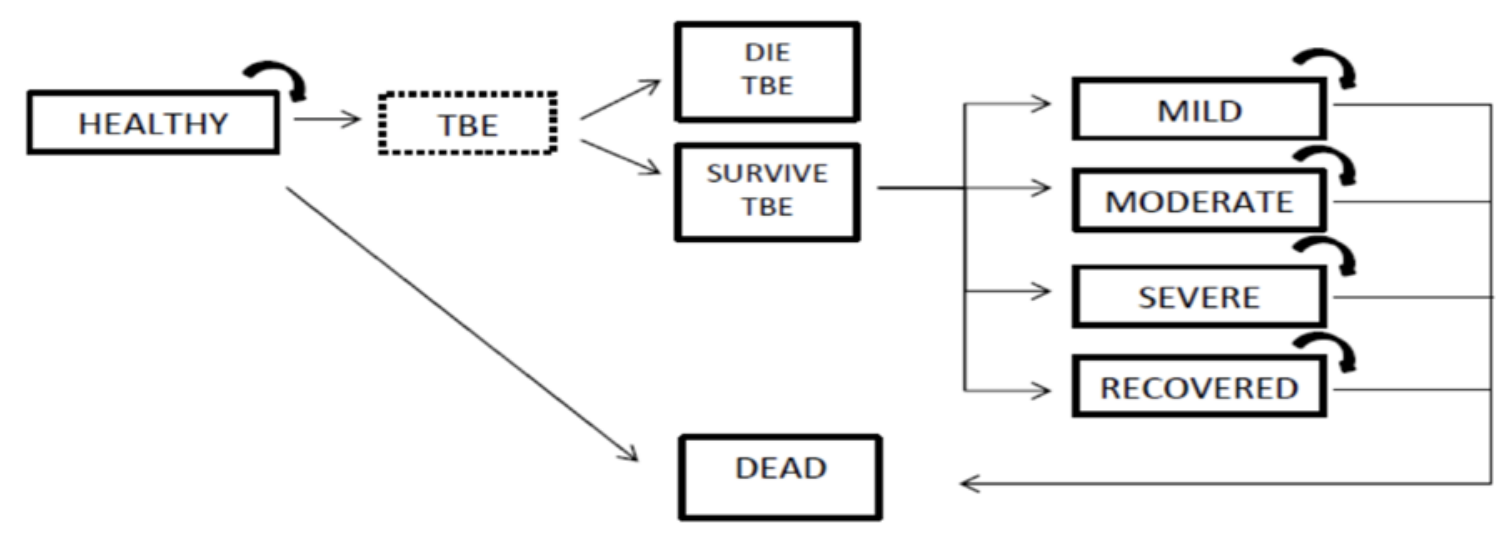

Figure 1 - simplified structure of the model with the different health states

Table 1- Values of the variables used in the economic analysis and

\section{variable}

value

Standard
error

range

$\begin{array}{ll}\text { (one-way source } & \\ \text { sensitivity } & \text { sours }\end{array}$

analysis)

Yearly incidence of TBE, by age groups

$1-5$

0.00003210 .000008025

6-10

$0.0000492 \quad 0.0000123$

$11-17$

$0.0000495 \quad 0.000012375$

$18-59$

$0.000078 \quad 0.0000195$

$60+$

$0.0001148 \quad 0.0000287$ 
Probability of sequelae by severity

Permanent mild neurological sequelae

$0.1 \quad 0.025$

Permanent moderate neurological

sequelae

$0.25 \quad 0.0625$

Permanent severe neurological

sequelae

$0.1 \quad 0.025$

$0.05-0.12$

Recovered and immune

remaining

Probability of death from disease 1st year, by age group

$1-18$

$19-40$

0

$41-60$

0

$>60$

$\begin{array}{lll}0.01 & 0.0025 & 0-0.03 \\ 0.02 & 0.005 & 0-0.03\end{array}$

$[1,6-12]$

Probability of death from disease

subsequent years, by age group

$1-18$

$19-40$

0

$41-60$

0

$>60$

0.01

0.0025

$0-0.03$

0.02

0.005

$0-0.03$

Efficacy of vaccine

0.987

0.002

$0.897-$

0.99

Costs of vaccine per dose (SEK), by age group

$<18$

180

45

$18+$

190

47.5

Direct cost per year (SEK)

Weighted average cost of

hospitalization in incident year, by age

group

$1-17$

$60717 \quad 15179$

$97534 \quad 24384$

$18-59$

$128982 \quad 32246$

Weighted average cost of

hospitalization in subsequent years, by

permanent state

Permanent mild neurological sequelae

Permanent moderate neurological

sequelae

2373

Permanent severe neurological

sequelae

9492

2373

4000

15000

$90000-$

$166632 \quad 41658 \quad 200000$

Average cost policlinic visits in incident year, by age group 
Average cost policlinic visits in

subsequent years, by permanent state

Permanent mild neurological sequelae

Permanent moderate neurological

sequelae

Permanent severe neurological

sequelae

Quality weights decrements

Incident year

Subsequent years, by permanent state

Permanent mild neurological sequelae

Permanent moderate neurological

sequelae

Permanent severe neurological

sequelae

Utility general population, by age group

1-9

$10-19$

20-29

30-39

40-49

$50-59$

$60-69$

$70-79$

80-89

90-99

Discount rate costs

Discount rate utility
$1230 \quad 308$

$4712 \quad 1178$

$6070 \quad 1518$

$1230 \quad 308$

$4712 \quad 1178$

$6070 \quad 1518$

[31]

$\begin{array}{lll}0.3 & 0.28 & 0.1-0.4\end{array}$

$\begin{array}{lll}0.18 & 0.24 & 0.05-0.3\end{array}$

$\begin{array}{lll}0.3 & 0.28 & 0.1-0.4\end{array}$

$\begin{array}{lll}0.54 & 0.29 & 0.2-0.6\end{array}$

0.89

0.89

0.89

0.88

0.86

0.83

0.8

0.79

0.74

0.74

0.03

0.03 
Table 2- Cost effectiveness results of base case analysis per person

\begin{tabular}{|c|c|c|c|c|c|c|c|}
\hline \multirow[b]{2}{*}{$\begin{array}{l}\text { Start } \\
\text { age }\end{array}$} & \multicolumn{3}{|c|}{ Mean costs (SEK) } & \multicolumn{3}{|c|}{ Mean QALYs } & \multirow{2}{*}{$\begin{array}{l}\text { Incremental } \\
\text { cost } \\
\text { effectiveness } \\
\text { ratio } \\
\text { (SEK/QALY) }\end{array}$} \\
\hline & $\begin{array}{c}\text { Vaccination } \\
\text { program }\end{array}$ & $\begin{array}{c}\text { No } \\
\text { Vaccination }\end{array}$ & Incremental & $\begin{array}{c}\text { Vaccination } \\
\text { program }\end{array}$ & $\begin{array}{c}\text { No } \\
\text { Vaccination }\end{array}$ & Incremental & \\
\hline 3 & 1113 & 869 & 244 & 25.954 & 25.945 & 0.009 & 27761 \\
\hline 40 & 1386 & 672 & 714 & 19.154 & 19.147 & 0.007 & 99527 \\
\hline 50 & 1439 & 562 & 877 & 16.112 & 16.107 & 0.005 & 160827 \\
\hline
\end{tabular}

SEK= Swedish Krona ; QALY= quality adjusted life years 


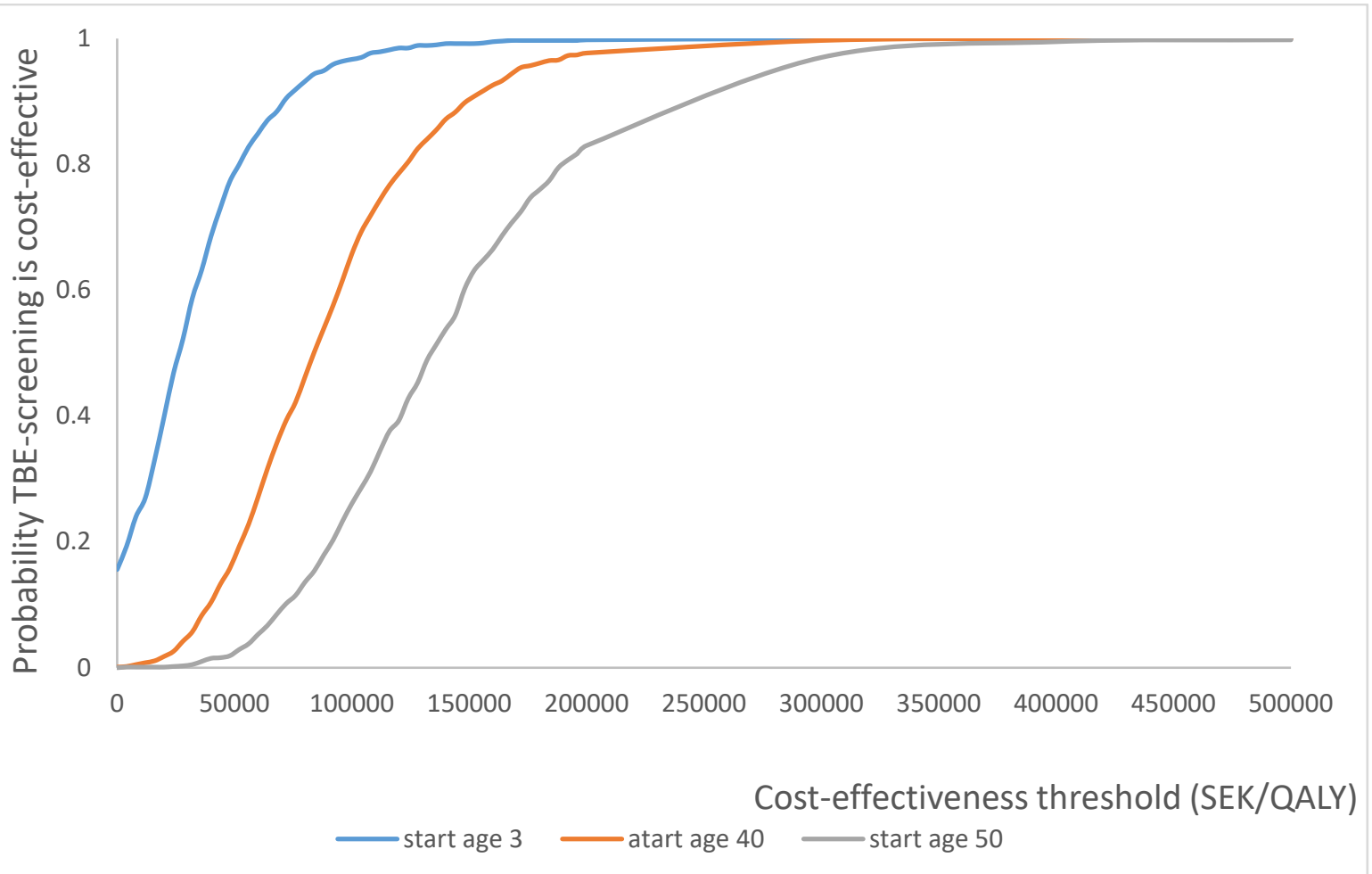

Figure 3 -Cost effectiveness acceptability curves for the TBE-vaccination program at different starting age. TBE= tick-born encephalitis; SEK= Swedish krona; QALY= qualityadjusted life years 


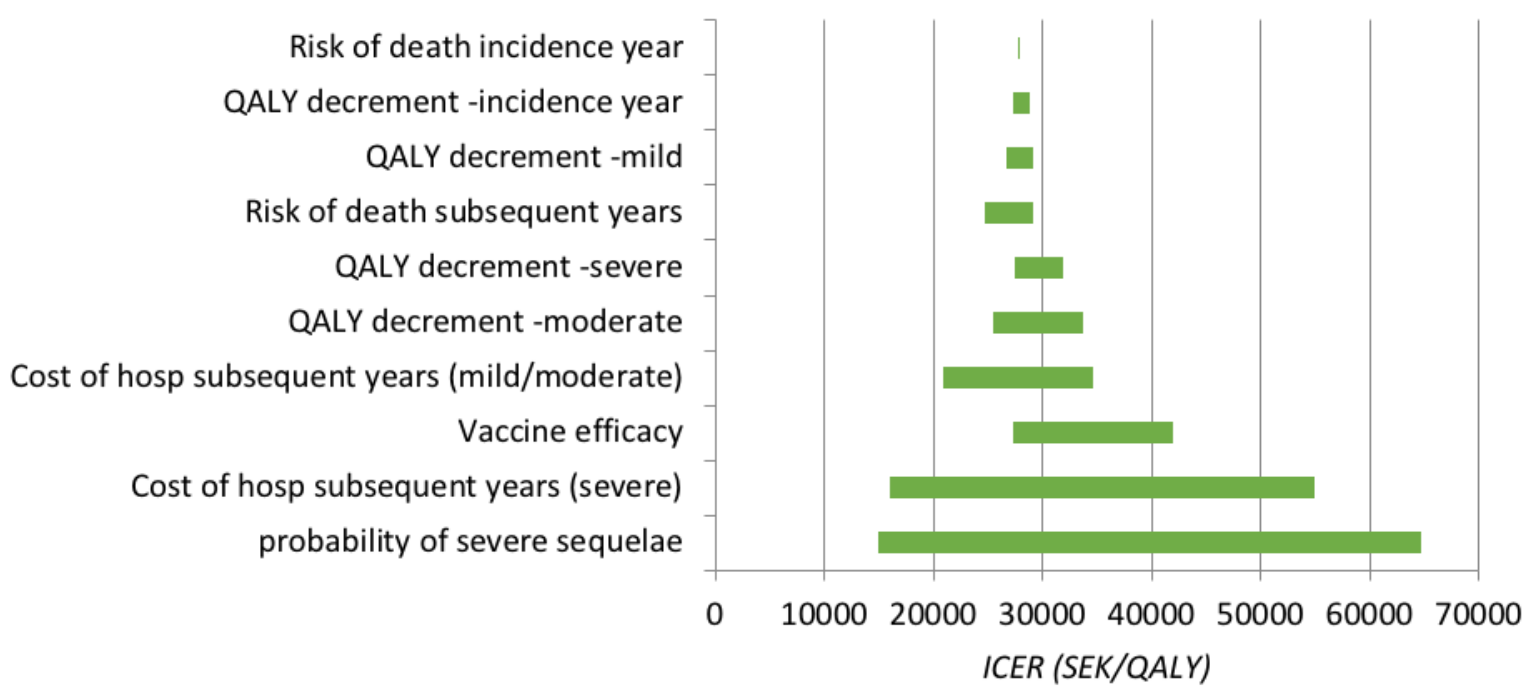

Figure 2- the one-way sensitivity analysis for the base-case analysis with start age 3. ICER= incremental cost effectiveness ratio 


\section{References}

[1] Lindquist L, Vapalahti O. Tick-borne encephalitis. Lancet 2008;371:1861-71. doi:10.1016/S0140-6736(08)60800-4.

[2] Kunze U. Report of the 19th Annual Meeting of the International Scientific Working Group on Tick-Borne Encephalitis (ISW-TBE) - TBE in a changing world. Ticks Tick Borne Dis 2017. doi:10.1016/j.ttbdis.2017.08.009.

[3] Clement J, Lagrou K, Saegeman V, Maes P, van Ranst M. the first tic-borne encephalitis case in the Netherlands: reflections and a note of caution. Euro Surveill 2016;21. doi:10.1099/vir.0.011437-0.

[4] Jääskeläinen A, Tonteri E, Pieninkeroinen I, Sironen T, Voutilainen L, Kuusi M, et al. Siberian subtype tick-borne encephalitis virus in Ixodes ricinus in a newly emerged focus, Finland. Ticks Tick Borne Dis 2016;7:216-23. doi:10.1016/j.ttbdis.2015.10.013.

[5] Jaenson TGT, Värv K, Fröjdman I, Jääskeläinen A, Rundgren K, Versteirt V, et al. First evidence of established populations of the taiga tick Ixodes persulcatus (Acari: Ixodidae) in Sweden. Parasites and Vectors 2016;9:1-8. doi:10.1186/s13071-0161658-3.

[6] Haglund M, Forsgren M, Lindh G, Lindquist L. A 10-year follow-up study of tickborne encephalitis in the Stockholm area and a review of the literature: Need for a vaccination strategy. Scand J Infect Dis 1996;28:217-24. doi:10.3109/00365549609027160.

[7] Haglund M, Günther G. Tick-borne encephalitis - Pathogenesis, clinical course and long-term follow-up. Vaccine 2003;21:2-9. doi:10.1016/S0264-410X(02)00811-3.

[8] Karelis G, Bormane A, Logina I, Lucenko I, Suna N, Krumina A, et al. Tick-borne 
encephalitis in Latvia 1973-2009: Epidemiology, clinical features and sequelae. Eur J Neurol 2012;19:62-8. doi:10.1111/j.1468-1331.2011.03434.x.

[9] Mickiene A, Laiskonis A, Günther G, Vene S, Lundkvist A, Lindquist L. Tickborne encephalitis in an area of high endemicity in lithuania: disease severity and long-term prognosis. Clin Infect Dis 2002;35:650-8. doi:10.1086/342059.

[10] Veje M, Nolskog P, Petzold M, Bergström T, Lindén T, Peker Y, et al. Tick-Borne Encephalitis sequelae at long-term follow-up: a self-reported case-control study. Acta Neurol Scand 2016;134:434-41. doi:10.1111/ane.12561.

[11] Bogovič P, Stupica D, Rojko T, Lotrič-Furlan S, Avšič-Županc T, Kastrin A, et al. The long-term outcome of tick-borne encephalitis in Central Europe. Ticks Tick Borne Dis 2017. doi:10.1016/j.ttbdis.2017.12.001.

[12] Günther G, Haglund M, Lindquist L, Forsgren M, Sköldenberg B. Tick-bone encephalitis in Sweden in relation to aseptic meningo-encephalitis of other etiology: a prospective study of clinical course and outcome. J Neurosci 1997;244:230-8.

[13] Loew-Baselli A, Konior R, Pavlova BG, Fritsch S, Poellabauer E, Maritsch F, et al. Safety and immunogenicity of the modified adult tick-borne encephalitis vaccine FSME-IMMUN®: Results of two large phase 3 clinical studies. Vaccine 2006;24:5256-63. doi:10.1016/j.vaccine.2006.03.061.

[14] Ehrlich HJ, Pavlova BG, Fritsch S, Poellabauer EM, Loew-Baselli A, ObermannSlupetzky O, et al. Randomized, phase II dose-finding studies of a modified tick-borne encephalitis vaccine: Evaluation of safety and immunogenicity. Vaccine 2003;22:217_ 23. doi:10.1016/S0264-410X(03)00563-2.

[15] World Health organisation. Vaccines against tick-borne encephalitis: WHO position paper 2011:241-56. doi:10.1186/1750-9378-2-15.Voir.

[16] Heinz FX, Stiasny K, Holzmann H, Grgic-Vitek M, Kriz B, Essl A, et al. Vaccination 
and tick-borne encephalitis, central Europe. Emerg Infect Dis 2013;19:69-76. doi:10.3201/eid1901.120458.

[17] Lindblom P, Wilhelmsson P, Fryland L, Matussek A, Haglund M, Sjöwall J, et al. Factors determining immunological response to vaccination against tick-borne encephalitis virus in older individuals. PLoS One 2014;9:1-10. doi:10.1371/journal.pone.0100860.

[18] Hertzell KB, Pauksens K, Rombo L, Knight A, Vene S, Askling HH. Tick-borne encephalitis (TBE) vaccine to medically immunosuppressed patients with rheumatoid arthritis: A prospective, open-label, multi-centre study. Vaccine 2016;34:650-5. doi:10.1016/j.vaccine.2015.12.029.

[19] Andersson CR, Vene S, Insulander M, Lindquist L, Lundkvist Å, Günther G. Vaccine failures after active immunisation against tick-borne encephalitis. Vaccine 2010;28:2827-31. doi:10.1016/j.vaccine.2010.02.001.

[20] public heath agency of Sweden. Tick Borne Encephalitis (TBE) 2017. https://www.folkhalsomyndigheten.se/folkhalsorapportering-statistik/statistikdatabaseroch-visualisering/sjukdomsstatistik/tick-borne-encephalitis-tbe/.

[21] Slunge D. The willingness to pay for vaccination against tick-borne encephalitis and implications for public health policy: Evidence from Sweden. PLoS One 2015;10:1-12. doi:10.1371/journal.pone.0143875.

[22] Stockholm County Council. TBE-vaccination n.d. http://www.vardgivarguiden.se/behandlingsstod/smittskydd/amnesomraden/vaccination /tbe.

[23] Askling HH, Insulander M, Hergens MP, Leval A. Tick borne encephalitis (TBE)vaccination coverage and analysis of variables associated with vaccination, Sweden. Vaccine 2015;33:4962-8. doi:10.1016/j.vaccine.2015.07.030. 
[24] Drummond M, Skulther M, Torrance G, O`brien B, Stoddart G. methods for economic evaluations of health care programmes. 3rd ed. New York: Oxford University Press (maker); 2005.

[25] TLV. Läkemedelsförmånsnämndens allmänna råd 2003:LFNAR 2003:2.

[26] Beran J, Lattanzi M, Xie F, Moraschini L, Galgani I. Second five-year follow-up after a booster vaccination against tick-borne encephalitis following different primary vaccination schedules demonstrates at least 10 years antibody persistence. Vaccine 2018. doi:10.1016/j.vaccine.2017.12.081.

[27] Konior R, Brzostek J, Poellabauer EM, Jiang Q, Harper L, Erber W. Seropersistence of TBE virus antibodies 10 years after first booster vaccination and response to a second booster vaccination with FSME-IMMUN 0.5 mL in adults. Vaccine 2017;35:3607-13. doi:10.1016/j.vaccine.2017.03.059.

[28] Bröker M, Schöndorf I. Are tick-borne encephalitis vaccines interchangeable? Expert Rev Vaccines 2006;5:461-6. doi:10.1586/14760584.5.4.461.

[29] Statistics Sweden. Dödstal per 1000 av medelfolkmängden efter ålder och kön. År 2000 - 20162017.

http://www.statistikdatabasen.scb.se/pxweb/sv/ssd/START_BE_BE0101_BE0101I/ Dodstal/?rxid=9e791d4b-2907-44cc-b19b-e720f5de8850.

[30] Stockholm County Council. Vaccines price list 2017:2017.

[31] Stockholm County Council. VAL database. Stockholm: 2014.

[32] Ghatnekar O, Steen Carlsson K. cost of illness of Stroke 2009. Stockholm: 2012.

[33] Stockholm County Council. folkhälsorapport. Stockholm: 2015.

[34] Ali M, MacIsaac R, Quinn TJ, Bath PM, Veenstra DL, Xu Y, et al. Dependency and health utilities in stroke: Data to inform cost-effectiveness analyses. Eur Stroke J 2017;2:70-6. doi:10.1177/2396987316683780. 
[35] Briggs,Andrew Claxton,Karl Sculpher M. Decision Modelling for Health Economic Evaluation. Oxford University Press (maker); 2006.

[36] Sörmland L. Hälsoekonomisk analys av allmän TBE-vaccinering av invånarna i Sörmland 2013.

[37] Šmit R. Cost-effectiveness of tick-borne encephalitis vaccination in Slovenian adults. Vaccine 2012;30:6301-6. doi:10.1016/j.vaccine.2012.07.083. 